\title{
Fatores relacionados à adesão às recomendações para administração de redose em cirurgias limpas
}

\author{
Factors related to adherence of recommendations for redose administration in clean surgeries
}

Factores relacionados a la adhesión a recomendaciones de administración de redosis en cirugías limpias

\section{Cristiane Schmitt ${ }^{1}$, Rúbia Aparecida Lacerda ${ }^{2}$}

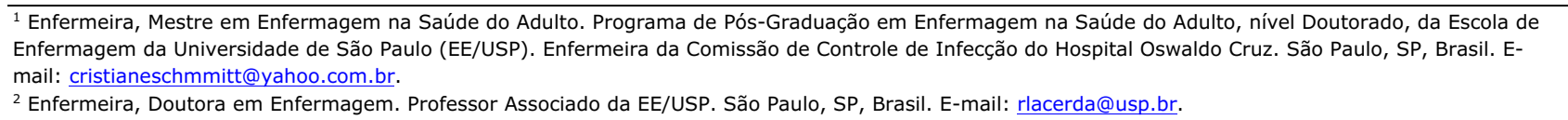

\section{RESUMO}

A ISC é um dos principais eventos adversos entre pacientes cirúrgicos e é responsável por 20\% das IRAS. A antibioticoprofilaxia cirúrgica é uma das práticas para prevenção de ISC, entretanto a adesão às diretrizes é baixa. Foi realizado um estudo observacional, transversal, retrospectivo que investigou o índice de adesão à redose e sua relação com características dos procedimentos e dos indivíduos. Foram avaliados 748 prontuários de cirurgias cardíacas, ortopédicas e neurológicas. Destes, 90 tinham indicação de redose, administrada em $26,6 \%$ das cirurgias. A adesão foi significativamente maior nas cirurgias cardíacas (34,3\%) e de maior IRIC. Concluiu-se que a adesão às diretrizes para administração de redose é baixa e é necessário atuar na melhoraria do processo de uso de antibioticoprofilaxia cirúrgica. $O$ uso de tecnologias aliado a conscientização, motivação das equipes e envolvimento da enfermagem podem ser alternativas.

Descritores: Antibioticoprofilaxia; Infecção Hospitalar; Segurança do Paciente; Cuidados de Enfermagem.

\section{ABSTRACT}

Surgical site infections (SSIs) are one of the main adverse events among surgical patients, being responsible for $20 \%$ of healthcare-associated infections (HAIs). Surgical antibiotic prophylaxis is one of the practices used to prevent SSIs, however, compliance with recommendations is low. A retrospective observational cross-sectional study investigated the index of compliance with redose and its relationship to characteristics of the procedures and individuals. A total of 748 records of cardiac, orthopedic and neurological surgeries were assessed. Of these, 90 had an indication of redose, which was administered in $26.6 \%$ of the surgeries. Compliance was significantly higher in cardiac surgeries (34.3\%) and those of greater SSI risk index. In conclusion, compliance with recommendations for redose administration is low and improving the process of surgical antibiotic prophylaxis use is needed. The use of technologies in association with awareness, motivation of the teams and involvement of the nursing staff may constitute alternatives.

Descriptors: Antibiotic Prophylaxis; Cross Infection; Patient Safety; Nursing Care.

\section{RESUMEN}

La ISC es uno de los principales efectos adversos entre pacientes quirúrgicos, responsable del $20 \%$ de las IRAs. La profilaxis antibiótica quirúrgica es una de las prácticas para la prevención de ISC, aunque con baja adhesión a sus directivas. Fue realizado un estudio observacional, transversal, retrospectivo, investigando el índice de adhesión a la redosis y su relación con características de procedimientos e individuos. Fueron evaluadas 748 historias clínicas de cirugías cardíacas, ortopédicas y neurológicas. De ellas, 90 tenían indicación de redosis, administrada en 26,6\% de las cirugías. La adhesión fue significativamente mayor en las cirugías cardíacas (34,3\%) y de mayor IRIC. Se concluyó en que la adhesión a las directivas para administración de redosis es baja, siendo necesario mejorar el proceso de profilaxis antibiótica quirúrgica. El uso de tecnologías en alianza con la concientización, motivación de los equipos y el involucramiento de la enfermería son también alternativas.

Descriptores: Profilaxis Antibiótica; Infección Hospitalaria; Seguridad del Paciente; Atención de Enfermería. 


\section{INTRODUÇÃO}

A segurança constitui um dos desafios da assistência à saúde em âmbito mundial. Em razão das mortes decorrentes de eventos adversos evitáveis ${ }^{(1)}$, em 2004 a Organização Mundial da Saúde (OMS) criou o projeto Aliança Mundial para a Segurança do Paciente, por meio do que foi denominado "Desafios Globais". O primeiro desafio está voltado à redução das Infecções Relacionadas à Assistência à Saúde (IRAS). O segundo, "Cirurgia Segura Salva Vidas", envolve ações para a redução de eventos adversos relacionados à cirurgia, incluindo, infecção do sítio cirúrgico (ISC) ${ }^{(2)}$.

O volume cirúrgico mundial é estimado em 234 milhões de procedimentos ao ano, com índices de complicações de até $16 \%$ nos países em desenvolvimento(3). As ISC figuram entre os principais eventos e são responsáveis por $20 \%$ de todas as IRAS ${ }^{(4-}$ ${ }^{6)}$, aumentam o tempo de internação em sete a $11 \operatorname{dias}^{(7)}$, dobram o risco de morte ${ }^{(8)}$ e tem custo anual estimado em 10 bilhões de dólares nos Estados Unidos ${ }^{(9)}$.

Paralelamente, a resistência microbiana é um problema de magnitude global, impactado pelas IRAS em consequência da prescrição de antimicrobianos em âmbito hospitalar, incluindo antibioticoprofilaxia cirúrgica ${ }^{(10)}$. Na Europa, entre 2011 e 2012, 35\% dos pacientes internados receberam pelo menos um antibiótico, 19,1\% indicados para o tratamento de IRAS. As prescrições para profilaxia cirúrgica corresponderam a $16,3 \%$ do total e foram mantidas por mais de um dia em mais da metade dos casos $(59,2 \%)^{(11)}$.

Globalmente, a redução dos índices de IRAS é necessária para a redução dos índices de resistência microbiana. Assim, ações para a redução das ISC são indispensáveis, considerando o volume cirúrgico mundial e o impacto destas sobre a incidência de IRAS. Melhorar os resultados cirúrgicos envolve atuação no pré, intra e pós-operatório, inclusive sobre as práticas de uso de antibioticoprofilaxia cirúrgica.

O uso apropriado de antibioticoprofilaxia cirúrgica está entre as práticas para prevenção de ISC e o cumprimento às recomendações reduz eventos adversos e efeitos relacionados à resistência microbiana(12-13). Entretanto, sua eficácia está atrelada ao cumprimento de todos os parâmetros de uso, incluindo o tipo de droga, dose, momento de início, duração e redose ou dose adicional em casos de procedimentos prolongados ${ }^{(12-13)}$. Não obstante, diversas publicações evidenciam baixa adesão às recomendações, sendo as maiores inadequações relacionadas momento de início e à duração(14-17).
Há poucas publicações abordando a conformidade quanto à administração de redose ${ }^{(18-22)}$. Possivelmente por não ser necessária à todas as cirurgias, mas somente àquelas cujas durações ultrapassam a meia vida da droga. Hipoteticamente, a adesão a esse parâmetro pode ser menor que aos demais, visto que a administração de redose ocorre durante o ato cirúrgico, o que poderia facilitar o esquecimento. Assim, é pertinente quantificar a adequação às diretrizes quanto à redose para que seja possível aplicar ações de melhoria que poderiam reduzir índices de ISC.

O presente estudo teve o objetivo de investigar o índice de adesão à redose em cirurgias cardíacas, neurológicas e ortopédicas, bem como sua relação com características dos procedimentos e dos indivíduos.

\section{MÉTODO}

Estudo observacional transversal retrospectivo, realizado em um hospital de 200 leitos, na cidade de São Paulo-SP, com média de 500 procedimentos cirúrgicos/mês. Os critérios de inclusão foram cirurgias limpas das especialidades cardíaca, ortopédica e neurológica, realizadas em pacientes adultos (18 anos e mais). Os critérios de exclusão foram pacientes em uso de antibioticoterapia e procedimentos não classificados como limpos. O cálculo amostral foi baseado no total de procedimentos dessas especialidades (7.404), realizados entre janeiro de 2005 e junho de 2009. Previu-se adesão de $50 \%$ às recomendações de uso de antibioticoprofilaxia cirúrgica, considerando-se margem de erro de $6 \%$ e poder amostral de $90 \%$, perfazendo 748 cirurgias, selecionadas de forma aleatória, por meio de sorteio dos registros dos pacientes utilizando-se uma tabela de números aleatórios.

As práticas referentes ao uso de antibioticoprofilaxia cirúrgica foram avaliadas com base nas diretrizes institucionais, as quais foram desenvolvidas em 2004, pelo Serviço de Controle de Infecção Hospitalar (SCIH), e aprovadas pela diretoria clínica. As diretrizes preconizam a administração de redose para procedimentos com duração maior ou igual a 180 minutos. Redose, ou dose adicional, foi definida como a administração de uma segunda dose de antibiótico antes do fechamento da ferida operatória.

Os dados foram obtidos de registros de prontuários. Foram consideradas apenas as cirurgias cuja droga administrada estava conforme a recomendação, uma vez que não havia padronização de tempo para administração de redose para drogas não relacionadas nas diretrizes da instituição. 
Os dados foram coletados entre novembro de 2009 e março de 2010. Os resultados encontrados foram correlacionados com o tipo de cirurgia, duração média do procedimento, idade e índice de risco cirúrgico (IRIC), o qual pode variar de zero a três, considerando-se a somatória do índice ASA, potencial de contaminação da ferida e duração do procedimento. Para as associações foram aplicados o Teste Qui Quadrado, Teste exato de Fisher ou Teste da Razão de Verossimilhança e Teste T para a comparação das médias. Adotou-se a de 0,05 para todos os testes.

O estudo foi orientado conforme descrito na Resolução no 196/1996 do Ministério da Saúde, autorizado formalmente pela instituição e submetido à aprovação do Comitê de Ética em Pesquisa da Escola de Enfermagem da Universidade de São Paulo (CEP-EEUSP), processo 824/2009-CEP-EEUSP. O modo de coleta de dados (em prontuários) não implicou em quaisquer riscos físicos ou psicológicos, e os resultados são apresentados de forma agregada, mantendo o anonimato dos sujeitos que compuseram a amostra.

\section{RESULTADOS}

Das 748 cirurgias, 252 (33,6\%) tiveram duração maior ou igual a 180 minutos, portanto, com indicação de redose. Destas, foram excluídas 90 cirurgias, por falta de dados, 68 por uso de antimicrobiano distinto do recomendado e quatro para as quais não foi identificada administração de droga profilática. Desse modo, foram consideradas na avaliação de adesão à redose, 90 cirurgias, sendo 45 neurológicas $(50,0 \%), 35$ cardíacas (38,9\%) e ortopédicas $10(11,1 \%)$ conforme apresentado na Tabela 1.

Tabela 1: Distribuição de procedimentos com indicação de redose segundo a especialidade. São Paulo, SP, Brasil, 2010.

\begin{tabular}{cccc}
\hline Especialidade & Cirurgias N (\%) & Indicação de redose N (\%) & Perdas N (\%) \\
\hline Cardíaca & $101(13,5)$ & $35(38,9)$ & $41(25,3)$ \\
Neurocirurgia & $128(17,1)$ & $45(50,0)$ & $48(29,6)$ \\
Ortopedia & $519(69,4)$ & $10(11,1)$ & $73(45,1)$ \\
\hline Total & $\mathbf{7 4 8 ( 1 0 0 , 0 )}$ & $\mathbf{9 0 ( 1 0 0 , 0 )}$ & $\mathbf{1 6 2 ( 1 0 0 , 0 )}$ \\
\hline
\end{tabular}

Das 90 cirurgias com indicação de redose, foi identificado registro de administração em apenas 24 $(26,6 \%)$ delas (Tabela 2$)$. Não houve diferença significativa quanto ao uso de redose e a idade média dos pacientes, gênero e duração dos procedimentos cirúrgicos, a despeito de supor-se maior número de doses adicionais entre procedimentos com maior duração.

Tabela 2: Características dos pacientes e procedimentos cirúrgicos conforme administração ou não de redoses de antibioticoprofilaxia durante cirurgias prolongadas. São Paulo, SP, Brasil, 2010.

\begin{tabular}{|c|c|c|c|}
\hline Características & & Redose & \\
\hline Variável & Sim & Não & $\mathbf{P}$ \\
\hline Idade (média, variação) & $53,34(30-73)$ & $50,46(25-80)$ & $0,3464^{\mathrm{a}}$ \\
\hline Duração do procedimento em minutos (média, variação) & $285,62(180-390)$ & $261,36(180-460)$ & $0,710^{\mathrm{a}}$ \\
\hline Gênero n (\%) & & & $0,674^{a}$ \\
\hline Feminino & $9(10,0)$ & $28(90,0)$ & \\
\hline Masculino & $15(28,3)$ & $38(71,7)$ & \\
\hline Especialidade $n(\%)$ & & & $0,027^{b}$ \\
\hline Cardíaca & $12(34,3)$ & $23(65,7 \%)$ & \\
\hline Neurológica & $12(26,7)$ & $33(73,3 \%)$ & \\
\hline Ortopédica & - & $10(100 \%)$ & \\
\hline Cardíaca n (\%) & & & $0,002^{b}$ \\
\hline RM & $10(41,7)$ & $14(58,3)$ & \\
\hline Outros* & - & $9(100,0)$ & \\
\hline Troca de válvula & $2(100,0)$ & - & \\
\hline Neurocirurgia n (\%) & & & LH $0,140^{b}$ \\
\hline Artrodese & $6(20,0)$ & $24(80,0)$ & \\
\hline Craniotomia & $6(46,2)$ & $7(53,8)$ & \\
\hline Laminectomia & - & $2(100,0)$ & \\
\hline Ortopedia n (\%) & & & Não se aplica \\
\hline Correção de fratura & - & $5(100,0)$ & \\
\hline Artroplastia de joelho & - & $2(100,0)$ & \\
\hline Artroplastia de quadril & - & $2(100,0)$ & \\
\hline IRIC n (\%) & & & $0,057^{b}$ \\
\hline 0 & $1(7)$ & $14(93,3)$ & \\
\hline 1 & $17(27,9)$ & $44(72,1)$ & \\
\hline 2 & $6(42,9)$ & $8(57,1)$ & \\
\hline
\end{tabular}


Ao comparar as especialidades cirúrgicas, observa-se que não foi administrada redose em cirurgias ortopédicas. A adesão foi significativamente maior entre procedimentos de cirurgia cardíaca (Tabela 2).

Em relação ao tipo de procedimento, houve indicação de redose, principalmente para artrodese de coluna, revascularização do miocárdio e correção de fratura. Entre as neurocirurgias, a proporção de redose foi maior entre as craniotomias, porém, sem significância estatística. Identificou-se diferença estatisticamente significativa entre as cirurgias cardíacas, com menor adesão nas revascularizações do miocárdio e outros procedimentos (uma ressecção de tumor, uma comissurotomia, três correções de aneurisma, três trocas de válvula associada a revascularização do miocárdio e uma correção de coartação de aorta), conforme apresentado na Tabela 2.

Em relação ao IRIC, $61(67,8 \%)$ pacientes foram classificados como IRIC-1, 15 (16,7\%) como IRIC-0 e 14 $(15,5 \%)$ como IRIC-2. A administração de redose foi mais frequente quanto maior o IRIC, com significância estatística (Tabela 2).

Os procedimentos classificados como IRIC-0 correspondem a artrodeses e revascularizações do miocárdio. Embora tais cirurgias não se enquadrem como prolongadas, segundo o ponto de corte do National Healthcare Safety Network (NHSN), tiveram duração maior do que 180 minutos, portanto, com indicação de redose, segundo o protocolo institucional.

\section{DISCUSSÃO}

As publicações disponíveis quanto a adesão às diretrizes de uso de antibioticoprofilaxia cirúrgica, em relação aos demais parâmetros, apresentam índices variando entre $4,9 \%$ e $25 \%{ }^{(14-17)}$. Em relação à redose, os índices de adesão variam entre $20 \%$ e $30 \%^{(18-22)}$. Na presente investigação a adesão à redose foi $26,6 \%$, ligeiramente maior que os índices de adesão aos demais parâmetros $^{(14-17)}$ de uso de antibioticoprofilaxia cirúrgica e semelhante à adesão à redose, segundo literatura disponível ${ }^{(18-22)}$. Assim, os resultados do presente estudo e das demais publicações ${ }^{(14-22)}$ refutam a hipótese inicial de que a adesão à redose seria menor quando comparada aos demais parâmetros de uso de antibioticoprofilaxia cirúrgica. Entretanto, evidenciam índices igualmente críticos.

Em relação ao IRIC, observou-se maior proporção de redoses quanto maior o IRIC, possivelmente, evidenciando maior preocupação da equipe com pacientes de maior risco cirúrgico. Quanto às especialidades cirúrgicas, houve pior adesão entre procedimentos neurológicos e ortopédicos quando comparados aos cardíacos.

Uma coorte retrospectiva, envolvendo 1.886 cirurgias cardíacas, com duração maior ou igual a 240 minutos, realizadas num período de 17 meses, evidenciou adesão de $30 \%$ às recomendações quanto a administração de redose $^{(19)}$. Não houve redução no risco para ocorrência de ISC com administração de doses adicionais durante o intraoperatório de forma geral. Entretanto, entre procedimentos com duração superior a 400 minutos o risco para ISC foi reduzido em $56 \%$, Odds Ratio 0,44 (IC $0,23-0,86)^{(19)}$. A evidência de maior risco para ISC entre procedimentos com maior duração deixa clara a importância da administração de redose, especialmente considerando a relação entre a duração e a complexidade das cirurgias.

0 índice de adesão à redose nessa coorte ${ }^{(19)}$ foi semelhante ao encontrado na presente investigação. Porém, não houve significância estatística em relação à adesão quando comparada com a duração dos procedimentos, ao contrário do referido estudo, cuja adesão foi maior entre procedimentos de maior duração. Em ambos também não houve diferenças estatisticamente significativas quanto a gênero e idade.

Um estudo multicêntrico, randomizado, envolvendo 29 hospitais e 4.472 cirurgias, (cardíacas, ortopédicas e ginecológicas) avaliou prospectivamente a associação entre o momento de administração da antibioticoprofilaxia cirúrgica e o uso de redose com o risco de ISC. Houve indicação de redose para 690 cirurgias, entretanto, apenas $21 \%$ dos pacientes receberam dose adicional o que pareceu reduzir o risco para infecção quando a dose inicial foi administrada no momento ideal (Odds ratio $=$ 3,08; IC $0,74-12,90)^{(21)}$.

Há poucos estudos disponíveis abordando adesão à redose na antibioticoprofilaxia cirúrgica. Dentre os existentes, a adesão às recomendações é muito baixa e nem todos evidenciaram associação entre redose e ISC, talvez em razão do pequeno número de cirurgias avaliadas $^{(18-22)}$. A despeito da falta de evidências para estabelecer a relação entre redose e ISC, se considerarmos a premissa de que o nível tissular de antimicrobianos deve ser mantido adequado durante todo o procedimento cirúrgico ${ }^{(12-13)}$ a baixa adesão à redose se apresenta como um problema relevante. Investigações sobre o assunto seriam úteis tanto para reconhecer a relação entre redose e ISC, quanto para fornecer subsídios para aumentar sua adesão.

Sobre este último aspecto, algumas estratégias já foram estudadas, entre elas, a introdução de um sistema 
eletrônico para sinalizar, ao anestesiologista, a necessidade de administração de redose. O alerta era dado 30 minutos antes do momento de infusão de redose para cirurgias cuja duração ultrapassava a meia vida da droga. Realizado em 2005, este estudo analisou dados de 148 procedimentos antes da implantação do sistema eletrônico e 139 após. Os resultados evidenciaram aumento de $20 \%$ para $58 \%$ na administração de redose após a introdução do sistema eletrônico. Após a introdução do alerta, dos procedimentos que não receberam redose a despeito de sua indicação, $20 \%$ tiveram o fechamento da ferida até 30 minutos após o anestesiologista receber o alerta eletrônico. Ou seja, estavam de acordo com as recomendações visto que não havia necessidade de dose adicional, uma vez que a ferida já havia sido fechada(18).

Outro estudo envolvendo 331 cirurgias cardíacas, com mais de 225 minutos de duração, também comparou - percentual de redoses administradas entre procedimentos com e sem o uso de alertas eletrônicos. Entre os procedimentos avaliados, 137 foram alocados no grupo intervenção (com alerta eletrônico) e 136 no grupo controle (sem alerta eletrônico). O uso de redose foi significativamente maior no grupo intervenção (68\%) do que no grupo controle ( $40 \%$ ), odds ratio 3,31 (IC $1,97-$ $5,56)$. O percentual de adesão à redose foi maior durante o período de estudo (40\%), mesmo entre cirurgias do grupo controle, quando comparado aos índices encontrados seis meses antes da intervenção (27\%). Este achado está, provavelmente, associado ao fato de que as equipes foram informadas sobre o estudo e, portanto, ficaram mais atentas à antibioticoprofilaxia cirúrgica. A incidência de ISC foi semelhante entre os grupos (6\% sem e $4 \%$ com alerta eletrônico), mas significantemente mais baixa do que no período anterior à intervenção (10\%; $p=0,02)^{(20)}$.

A eficácia de dois diferentes tipos de alertas eletrônicos para a administração de redose foi avaliada em um hospital universitário. O primeiro fazia parte do sistema informatizado utilizado pelos anestesiologistas e emitia alertas em um intervalo previamente definido. $O$ fato do sistema em uso emitir alertas mesmo sem a administração da primeira dose do antibiótico e não possibilitar a inserção de tempos distintos conforme o tipo de droga levou ao desenvolvimento uma nova alternativa capaz de identificar se a antibioticoprofilaxia foi administrada e qual foi a droga utilizada. Com base nessas informações, o novo sistema emitia alertas repetidos a cada seis minutos, conforme o tipo de droga usada. Foram avaliados 375 procedimentos com indicação de redose,
188 com o sistema existente e 187 com o novo. O índice de adesão à redose aumentou de $62,5 \%$ para $83,9 \%$ $(p<0.001)^{(22)}$.

Esses três estudos ${ }^{(20-22)}$ demonstraram que o uso de recursos eletrônicos propicia melhor adesão às recomendações quanto à administração de redose, e possível redução do risco para ocorrência de ISC. Contudo, há que se questionar por que, apesar de recursos tão evidentes, é dada tão pouca atenção à redose, visto que se trata de um erro que pode impactar negativamente o resultado dos tratamentos cirúrgicos. Entretanto, tal aspecto não foi discutido nesses estudos ${ }^{(20-}$ 22).

O check list proposto pela OMS envolve a participação de toda a equipe cirúrgica, inclusive enfermeiros, e propõe a verificação de itens de segurança antes da anestesia, antes da incisão e antes do paciente deixar a sala cirúrgica. Contudo, em relação ao uso de antibioticoprofilaxia cirúrgica é recomendada apenas a checagem da administração da primeira dose, até 60 minutos antes da incisão. A administração de redose, não é contemplada no documento original ${ }^{(2)}$.

A administração de antibioticoprofilaxia cirúrgica, geralmente, está sob responsabilidade do anestesiologista. Porém, aquiescendo com as considerações da OMS, sobre a relevância do trabalho em equipe, a responsabilidade sobre tal prática deve ser multiprofissional. A melhoria das práticas, mediante atuação multiprofissional, é evidenciada pelos resultados de um estudo envolvendo cesarianas. Após o envolvimento de enfermeiras no processo, a adesão à administração de antibioticoprofilaxia aumentou de $25 \%$ para $100 \%$, com redução significativa dos índices de infecção. Os autores desse estudo argumentam que, em virtude da assistência direta aos pacientes, a enfermagem encontra-se em posição privilegiada para detectar falhas de medicação e assim, atuar na prevenção de eventos adversos o que poderia ser útil em se tratando da administração de redose para procedimentos prolongados $^{(23)}$.

A despeito deste estudo(23) não tratar de redose, confere situação semelhante, pois trata do esquecimento da administração da antibioticoprofilaxia cirúrgica em obstetrícia. Resgata, ainda, a relevância do elemento humano, neste caso, a histórica participação e valorização da enfermagem em ações de segurança ao paciente na assistência à saúde. Quanto à antibioticoprofilaxia cirúrgica, embora a decisão sobre o uso seja atributo médico, a garantia da adesão, inclusive da redose, pode ser compartilhada pela ação de outros profissionais. 


\section{CONCLUSÃO}

O presente estudo identificou baixo percentual de adesão à aplicação de redose em cirurgias prolongadas, sendo maior entre cirurgias cardíacas e entre aquelas de maior IRIC. Apesar da escassa literatura sobre essa temática, os resultados obtidos inserem-se na variação encontrada nas demais investigações realizadas, evidenciando que a adesão às recomendações de uso de antibioticoprofilaxia cirúrgica não é um problema observado apenas em países em desenvolvimento, mas em âmbito mundial. O mapeamento dos índices de adesão às diretrizes para o uso de antibioticoprofilaxia cirúrgica nos permite ter dimensão do problema e, desse modo, propor ações para a melhoria do processo.

Assim como no presente estudo, em algumas das publicações citadas, a coleta de dados foi retrospectiva, o que pode ser tratado como uma limitação. Essa metodologia pode ter subestimado a adesão à administração de redose, uma vez que é possível que o

\section{REFERÊNCIAS}

1. Stelfox HT, Palmisani S, Scurlock C, Orav EJ, Bates DW. The "To Err is Human" report and the patient safety literature. Qual Saf Health Care [Internet]. 2006 [acesso em: 31 mar 2015];15(3):174-8. Disponível em:

http://dx.doi.org/10.1136/qshc.2006.017947.

2. World Health Organization. WHO guidelines for safe surgery 2009 [Internet]. Geneva: World Health Organization; 2009 [acesso em: 31 mar 2015]. Disponível em:

http://whqlibdoc.who.int/publications/2009/9789241598552_eng .pdf.

3. Weiser TG, Regenbogen SE, Thompson KD, Haynes AB, Lipsitz $S R$, Berry WR et al. An estimation of the global volume of surgery: a modelling strategy based on available data. Lancet [Internet]. 2008 [acesso em: 31 mar 2015];372(9633):139-44. Disponível em: http://dx.doi.org/10.1016/S0140-

6736(08)60878-8.

4. Klevens MR, Edwards JR, Richards Jr CL, Horan TC, Gaynes $\mathrm{RP}$, Pollock DA et al. Estimating health care-associated infections and deaths in U.S hospitals, 2002. Public Health Rep [Internet]. 2007 [acesso em: 31 mar 2015];122(2):160-6. Disponível em: http://www.ncbi.nlm.nih.gov/pmc/articles/PMC1820440/. 5. Lissovoy G, Fraeman K, Hutchins V, Murphy D, Song D, Vaughn BB. Surgical site infection: incidence and impact on hospital utilization and treatment costs. Am J Infect Control [Internet]. 2009 [acesso em: 31 mar 2015];37(5):387-97. Disponível em: http://dx.doi.org/10.1016/j.ajic.2008.12.010. 6. European Centre for Disease Prevention and Control. Surveillance of surgical site infections in Europe 2010-2011 [Internet]. Stockholm: ECDC; 2013 [acesso em: 31 mar 2015]. Disponível em:

http://ecdc.europa.eu/en/publications/Publications/SSI-ineurope-2010-2011.pdf.

7. Zimlichman E, Henderson D, Tamir O, Franz C, Song P, Yamin $\mathrm{CK}$ et al. Health care-associated infections: a meta-analysis of costs and financial impact on the US health care system. JAMA Intern Med [Internet]. 2013 [acesso em: 31 mar 2015];173(22):2039-46. Disponível em:

http://dx.doi.org/10.1001/jamainternmed.2013.9763. antibiótico tenha sido administrado, mas não registrado em prontuário.

É necessário atuar sobre o processo de uso de antibioticoprofilaxia cirúrgica. Ações em tempo real podem aumentar consistentemente a adesão às diretrizes, incluindo redose. Entretanto, apenas o uso de tecnologias não é suficiente para sanar o problema. É indispensável atuar diretamente sobre o elemento humano e, para tanto, há que se investir na conscientização e motivação das equipes, uma vez que o problema não se situa exclusivamente sobre a falta de conhecimento. Envolver a equipe na construção das diretrizes, apresentar resultados de desempenho quanto à adesão às diretrizes de uso de antibioticoprofilaxia cirúrgica e taxas de ISC, podem ser alternativas a se somar à tecnologia disponível para obtenção de melhores resultados. A segurança em cirurgia implica em coordenação conjunta da equipe e a enfermagem tem um papel importante neste aspecto. Sua participação no processo de uso de antibioticoprofilaxia cirúrgica pode contribuir para melhorar a adesão ao uso de redose em cirurgias prolongadas.

8. Kirkland KB, Briggs JP, Trivette SL, Wilkinson WE, Sexton DJ. The impact of surgical-site infections in the 1990s: attributable mortality, excess length of hospitalization, and extra costs. Infect Control Hosp Epidemiol. 1999;20(11):725-30.

9. Scott DR. The Direct Medical Costs of Healthcare-Associated Infections in U.S. Hospitals and the Benefits of Prevention [Internet]. Atlanta: CDC; 2009 [acesso em: 31 mar 2015]. Disponível em:

http://www.cdc.gov/hai/pdfs/hai/scott_costpaper.pdf. 10. World Health Organization. The evolving threat of antimicrobial resistance: options for action [Internet]. Geneva: WHO; 2012 [acesso em: 31 mar 2015]. Disponível em: http://whqlibdoc.who.int/publications/2012/9789241503181_eng .pdf.

11. European Centre for Disease Prevention and Control. Point prevalence survey of healthcare- associated infections and antimicrobial use in European acute care hospitals [Internet]. Stockholm: ECDC; 2013 [acesso em: 31 mar 2015]. Disponível em:

http://ecdc.europa.eu/en/publications/Publications/healthcareassociated-infections-antimicrobial-use-PPS.pdf.

12. Scottish Intercollegiate Guidelines Network. SIGN 104 Antibiotic prophylaxis in surgery: a national clinical guideline (july 2008, updated April 2014). Edinburgh: SIGN; 2008 [acesso em: 31 mar 2015]. Disponível em:

http://www.sign.ac.uk/pdf/sign104.pdf.

13. Bratzler DW, Dellinger EP, Olsen KM, Perl TM, Auwaerter PG, Bolon $\mathrm{MK}$ et al. Clinical practice guidelines for antimicrobial prophylaxis in surgery. Am J Health Syst Pharm [Internet]. 2013 [acesso em: 31 mar 2015];70(3):195-283; Disponível em: http://dx.doi.org/10.2146/ajhp120568.

14. Lallemand S, Thouverez M, Bailly P, Bertrand X, Talon D. Non-observance of guidelines for surgical antimicrobial prophylaxis and surgical-site infections. Pharm World Sci [Internet]. 2002 [acesso em: 31 mar 2015];24(3):95-9. Disponível em: http://dx.doi.org/10.1023/A:1016122202439. 15. Van Kasteren ME, Mannien J, Kullberg BJ, Boer AS, Nagelkerke NJ, Ridderhof $M$ et al. Quality improvement of surgical prophylaxis in Dutch hospitals: evaluation of a multi-site intervention by time series analysis. J Antimicrob Chemother 
[Internet]. 2005 [acesso em: 31 mar 2015];56(6):1094-102. Disponível em: http://dx.doi.org/10.1093/jac/dki374.

16. Miliani K, L'Hériteau F, Astagneau P. Non-compliance with recommendations for the practice of antibiotic prophylaxis and risk of surgical site infection: results of a multilevel analysis from the INCISO Surveillance Network. J Antimicrob Chemother [Internet]. 2009 [acesso em: 31 mar 2015];64(6):1307-15. Disponível em: http://dx.doi.org/10.1093/jac/dkp367. 17. Schmitt C, Lacerda RA, Padoveze MC, Turrini RN. Applying validated quality indicators to surgical antibiotic prophylaxis in a Brazilian hospital: learning what should be learned. Am J Infect Control [Internet]. 2012 [acesso em: 31 mar 2015];40(10):9602. Disponível em: http://dx.doi.org/10.1016/j.ajic.2012.01.016. 18. St Jacques P, Sanders N, Patel N, Talbot TR, Deshpande JK, Higgins M. Improving timely surgical antibiotic prophylaxis redosing administration using computerized record prompts. Surg Infect (Larchmt) [Internet]. 2005 [acesso em: 31 mar 2015];6(2):215-21. Disponível em:

http://dx.doi.org/10.1089/sur.2005.6.215.

19. Zanetti G, Giardina R, Platt R. Intraoperative redosing of cefazolin and risk for surgical site infection in cardiac surgery. Emerg Infect Dis [Internet]. 2001 [acesso em: $31 \mathrm{mar}$ 2015];7(5):828-31. Disponível em: http://dx.doi.org/10.3201/eid0705.017509.

20. Zanetti G, Flanagan HL Jr, Cohn LH, Giardina R, Platt R. Improvement of intraoperative antibiotic prophylaxis in prolonged cardiac surgery by automated alerts in the operating room. Infect Control Hosp Epidemiol. 2003;24(1):13-6.

21. Steinberg JP, Braun BI, Hellinger WC, Kusek L, Bozikis MR, Bush AJ et al. Timing of antimicrobial prophylaxis and the risk of surgical site infections: results from the Trial to Reduce Antimicrobial Prophylaxis Errors. Ann Surg [Internet]. 2009 [acesso em: 31 mar 2015];250(1):10-6. Disponível em: http://dx.doi.org/10.1097/SLA.0b013e3181ad5fca.

22. Nair BG, Newman SF, Peterson GN, Schwid HA. Automated electronic reminders to improve redosing of antibiotics during surgical cases: comparison of two approaches. Surg Infect (Larchmt) [Internet]. 2011 [acesso em: 31 mar 2015];12(1):5763. Disponível em: http://dx.doi.org/10.1089/sur.2010.047. 23. Shimoni Z, Kama N, Mamet Y, Glick J, Dusseldorp N, Froom P. Empowering surgical nurses improves compliance rates for antibiotic prophylaxis after caesarean birth. J Adv Nurs

[Internet]. 2009 [acesso em: 31 mar 2015];65(11):2345-9.

Disponível em: http://dx.doi.org/10.1111/j.1365-

2648.2009.05096.x.

Artigo recebido em 19/02/2013.

Aprovado para publicação em 30/05/2014.

Artigo publicado em 31/03/2015. 